\title{
Las experiencias de incertidumbre de jóvenes mexicanos en tratamiento de diálisis peritoneal
}

\author{
Blanca Alejandra Díaz-Medina' ${ }^{1}$, Denise Guerreiro-Vieira-da-Silva² \\ ${ }^{1}$ Universidad del Valle de México. Escuela de Ciencias de la Salud. Campus Zapopan. México \\ 2 Universidad Federal de Santa Catarina. Brasil
}

Como citar este artículo:

Díaz-Medina BA, Guerreiro-Vieira-da-Silva D. Las experiencias de incertidumbre de jóvenes mexicanos en tratamiento de diálisis peritoneal.

Enferm Nefrol. 2020 Abr-Jun;23(2):160-7

\section{Resumen}

Objetivo: Describir las experiencias de incertidumbre de jóvenes con insuficiencia renal que viven en tratamiento dialítico.

Material y Método: Estudio etnográfico en Guadalajara, México. Participaron 12 jóvenes con insuficiencia renal y en diálisis peritoneal. Se realizaron entrevistas narrativas y observación participante. Se hizo análisis hermenéutico.

Resultados: Se identificaron dos modalidades de incertidumbre, la personal y la médica. Las fuentes de la personal fueron los cambios corporales, la reconfiguración identitaria y de roles, la falta de recursos materiales y al pensar en los proyectos a futuro. Las fuentes de la médica fueron falta de información, el inicio y el manejo del tratamiento y el progreso de la enfermedad.

Conclusiones: La incertidumbre se vuelve una constante en sus vidas y su manejo se ve influenciado por los recursos materiales y los apoyos sociales que reciben.

PALABRAS CLAVE: experiencia; incertidumbre; enfermedad renal; diálisis peritoneal.

\section{Correspondencia:}

Blanca Alejandra Díaz Medina

Email: blankmusic87@gmail.com

\section{Uncertainty experiences of young Mexicans in peritoneal dialysis treatment}

\section{Abstract}

Objective: To describe the uncertainty experiences of young people with renal failure with dialysis treatment.

Material and Method: Ethnographic study in Guadalajara, Mexico. Twelve young people with renal failure and on peritoneal dialysis participated. Narrative interviews and participant observation were conducted. Hermeneutical analysis was performed.

Results: Two types of uncertainty were identified: personal and medical. The sources of personal uncertainty were bodily changes, identity and role reconfiguration, lack of material resources and when thinking about future projects. The sources of medical uncertainty were lack of information, the initiation and management of treatment, and the progress of the disease.

Conclusions: Uncertainty becomes a constant in the patients' lives and management is influenced by the material resources and social supports they receive.

KEYWORDS: Experience; Uncertainty; Kidney disease; Peritoneal dialysis. 


\section{Introducción}

Las enfermedades crónicas han ido en aumento en las últimas décadas, tanto en prevalencia como en incidencia. Entre ellas destaca la enfermedad renal crónica $(E R C)$ que se ha convertido en un problema de salud pública en numerosos países ${ }^{1}$, siendo una de las diez principales causas de muerte en México². Se considera una enfermedad que genera gastos catastróficos ${ }^{3}$ debido a los altos costos de las terapias de reemplazo renal. En México, más de 100 mil ciudadanos utilizan alguna de estas terapias, principalmente la diálisis peritoneal. En el caso de Latinoamérica, el $73 \%$ de la población utiliza esa terapia dialítica4.

Para los jóvenes, con ERC, sus proyectos de vida e ideales se ven interrumpidos por un padecimiento que no tiene cura ${ }^{5-7}$. En particular, aquellos que están en diálisis peritoneal continua ambulatoria (DPCA), se enfrentan a un catéter permanente en el abdomen y a un tratamiento que deben de llevar a cabo cada seis horas en casa. $Y$ aunque es considerada la terapia dialítica de menor costo, para quienes no cuentan con seguridad social es difícil mantener el tratamiento ${ }^{8}$. Sin embargo, dentro del área de la salud, tradicionalmente se ha utilizado el modelo biomédico para analizar a la enfermedad, usando el método y conocimiento científico para obtener un diagnóstico y proponer un tratamiento, dejando de lado las experiencias sobre el padecimiento y cómo otros factores, como son lo emocional, social y económico también afectan a las personas?.

En este marco, donde desarrollan una enfermedad crónica que cambia sus vidas y conlleva un tratamiento de alto costo, se configura un escenario de incertidumbre. En las últimas décadas, se ha reconocido a la incertidumbre como una dimensión importante de la experiencia del padecimiento $0^{10,11}$, sobre todo entre quienes padecen enfermedades crónicas. Su importancia radica en querer comprender las causas y efectos que tiene sobre las personas enfermas, puesto que se reconoce que puede llevarlos a la inestabilidad emocional, física y sociall1.

La incertidumbre es un estado cognitivo en el cual no se puede determinar el significado o el valor de un evento y/u objeto, ni predecir con exactitud los resultados ${ }^{11}$. Las personas con enfermedades crónicas experimentan incertidumbre desde el momento que aparecen los primeros síntomas de la enfermedad, hasta que llevan a cabo el tratamiento y seguimiento de la misma9. Por lo tanto, las experiencias de incertidumbre se vuelven un fenó- meno generalizado, que está mediado por sentimientos de confianza y control, que se tienen sobre situaciones específicas o globales ${ }^{12}$. En este trabajo interesa, más que medir los niveles de incertidumbre, comprender sus experiencias en relación a ella, qué es lo que la causa, cómo la experimentan y cómo la manejan.

No obstante, es un tema poco estudiado, sobre todo en población con ERC en DPCA ${ }^{13}$. Sin embargo, hay estudios que evidencian las preocupaciones e incertezas, de esta población, ante la posibilidad de padecer una infección y el no tener control sobre su cuerpo $0^{6,14,15}$. Reportan que desarrollan una nueva identidad, a partir de la enfermedad, lo que les causa momentos de crisis ${ }^{13,16}$. Asimismo, sufren pérdida de la cotidianidad, porque su vida cambia totalmente y no pueden hacer planes a futuro $0^{6,13}$

Por todo lo anterior, el objetivo de este trabajo es comprender las experiencias de incertidumbre que viven los jóvenes que utilizan el tratamiento de DPCA. Se espera que los resultados contribuyan en las decisiones que toman los profesionales de la salud en relación con el cuidado al paciente, brindando nuevos elementos que amplíen la comprensión de las dificultades cotidianas de los jóvenes con ERC, las situaciones o condiciones que promueven la incertidumbre y cómo las manejan.

\section{Materiales y métodos}

Se llevó a cabo un estudio cualitativo, desde una perspectiva critico interpretativa ${ }^{17}$, en la Zona Metropolitana de Guadalajara, Jalisco, México. Dicha zona concentra seis municipios centrales del estado, y en ellos a los hospitales públicos más importantes en esta región que dan atención especializada a la población con ERC. Se solicitó apoyo a dos asociaciones civiles que trabajan con personas con enfermedad renal, para contactar a posibles participantes y a partir de un muestreo por bola de nieve, contactar a otros. Los criterios de inclusión es que fueran jóvenes que hayan desarrollado enfermedad renal desde la niñez o adolescencia y que actualmente estén en tratamiento de DPCA. Al inicio de la investigación no se tenía decidido el tamaño de la muestra, sin embargo, con base a la revisión de la literatura de otras investigaciones de diseño cualitativo, se consideró incluir al menos 10 participantes. De las 18 personas contactadas, 12 aceptaron participar en el estudio. Siete fueron hombres y cinco mujeres, de entre 18 y 30 años. Al analizar la información obtenida se consideró que era un tamaño de muestra adecuado 
debido a la riqueza de los datos que permiten responder a las preguntas de investigación.

El trabajo de campo consistió en observaciones y entrevistas durante 19 meses, entre los años 2013 y 2015. Se hizo observación no participante en las clínicas donde son atendidos y se realizaron notas descriptivas sobre esos espacios y las interacciones entre los pacientes y profesionales de la salud. También se hizo observación participante en las casas y las asociaciones civiles que los apoyan, donde también se elaboraron notas de campo para describir el lugar, alguna actividad o un tema del que se habló, formal o informalmente. Para profundizar en las experiencias de incertidumbre, se hicieron entrevistas narrativas que permitieran que ellos contaran sus historias de vida sobre el padecimiento. Se inició con la pregunta ¿Cómo es que te diagnostican la enfermedad? $Y$ a partir de ahí se exploraron sus experiencias de incertidumbre. Y, por último, se tuvo conversaciones con los participantes por medio de los chats de Facebook y WhatsApp. Se tomó esta decisión debido a que la mayoría hacen uso de estos medios y han formado parte de la cotidianidad en la vida de las personas.

Las entrevistas se transcribieron y se almacenaron en el software Ethnograph v6, junto con las notas de campo y las conversaciones de los chats. Se hizo análisis hermenéutico ${ }^{18}$, dividiéndolo en tres fases: la comprensión ingenua destinada a adquirir el sentido del todo; el análisis estructural que se basó en un análisis temático y; la interpretación comprehensiva, en la cual los conocimientos previos, la comprensión ingenua y el análisis estructural se relacionan para una nueva comprensión. A partir de ello se identificaron los temas principales sobre las fuentes de incertidumbre y posteriormente se agruparon en la modalidad de incertidumbre correspondiente, fuese personal, médica o social ${ }^{19,20}$.

El proyecto fue evaluado por un comité académico de la Universidad de Guadalajara, posteriormente se presentó de forma verbal a todos los participantes. Aclaradas las dudas se les invitó a participar y se les solicitó su consentimiento informado. Además, se emplearon seudónimos para asegurarles el anonimato.

\section{Resultados}

Los jóvenes que usan la DPCA experimentan principalmente dos modalidades de incertidumbre, la personal y la médica. En la Tabla 1 se identifican las fuentes correspondientes de ambas modalidades.
Tabla 1. Fuentes de incertidumbre en los jóvenes con enfermedad renal crónica en diálisis peritoneal.

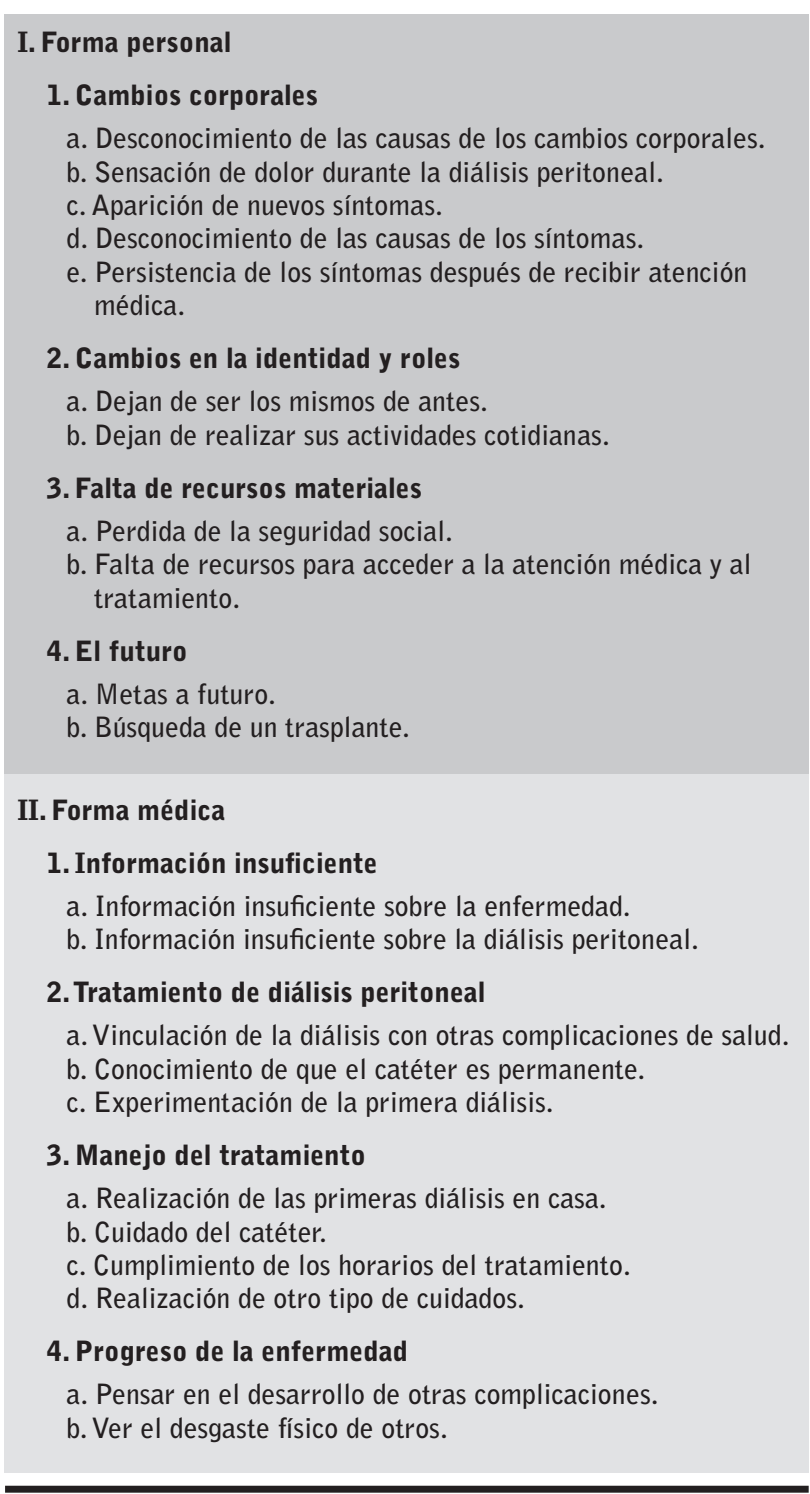

\section{La incertidumbre personal}

\section{Los cambios corporales}

El cuerpo juega un papel central en el tratamiento ya que les alerta sobre el estado en el que se encuentran. Aprenden a reconocer cualquier cambio en sus sensaciones y buscan explicaciones al respecto. Los continuos cambios que experimentan es un indicio de que su cuerpo se ha vuelto impredecible y no tienen control sobre el mismo, lo cual se complejiza al no saber la causa, ni qué esperar en el futuro.

Al inicio de la enfermedad experimentan cambios corporales, tales como dolores de cabeza, vómitos, can- 
sancio e hinchazón. Algunos perciben los cambios gradualmente, pero en otros aparecen repentinamente. Tal situación les genera zozobra por lo que preguntan qué está pasando, buscan alguna explicación y los hace reaccionar con preocupación. Antonio se refiere a ello:

"Se te acumula como desesperación. Decir 'tengo tantas ganas de hacer cosas, pero no puedo; mi cuerpo no reacciona, mi cuerpo no me da ese empujón que necesito para hacerlo... ¿Por qué si tengo tantas ideas, tantas ganas de hacer cosas... mi cuerpo no me deja?, ¿... ¿Por qué soy tan flojo?"

Los jóvenes manejan de diversas formas la incertidumbre por los cambios corporales. Principalmente buscan información con los profesionales de la salud, sus pares o las asociaciones civiles. También por medio del Internet, especialmente en las redes sociales virtuales, allí suelen preguntar sobre el tema o pedirles alguna recomendación. Si perciben que las indicaciones médicas no son suficientes se refugian en su religión y/o tratamientos alternativos.

\section{Los cambios en la identidad y roles}

Los jóvenes también perciben cambios en su identidad y en los roles que desempeñan. Piensan que no van a ser los mismos ni realizar las actividades que solían hacer. Por ello no tienen certeza de quienes son, que harán con sus vidas o si será algo temporal.

A pesar de los esfuerzos que realizan para retomar la vida que tenían en el pasado, cada vez les es más evidente que necesitan aceptar su padecimiento y la identidad de personas enfermas. De igual forma, al enfrentar dificultades para retomar sus estudios o trabajo no saben si lograrán cumplir sus proyectos personales. Por ejemplo, para Beatriz ha sido difícil retomar su profesión y no sabe si logrará realizar sus metas personales:

"(La enfermedad) me cambió la vida, siempre he tratado de recuperar lo que era, o el plan de vida que tenía y... (llora) no lo he logrado... Me siento muy agobiada, muy cansada. Ha sido mucho tiempo y si te soy sincera a veces he llegado a pensar que no voy a salir de esto. 0 sea, que voy a vivir así hasta lo que dure dializándome y realmente no sé".

Para manejar este tipo de incertidumbre, dicen que es importante mantener una buena actitud ante las dificultades y sobre todo aceptar la enfermedad. Además, buscan estrategias para seguir haciendo lo que les gusta, retomar los estudios o trabajo.
La esperanza de obtener un trasplante es de gran importancia, ya que piensan que con eso volverán a ser como eran antes y retomarán sus proyectos de vida.

\section{Falta de recursos materiales}

La DPCA es considerada la terapia de más bajo costo. A pesar de ello, los participantes no suelen contar con los recursos financieros necesarios para llevarla a cabo, lo que los hace sentir preocupados ante la posibilidad de dejar de dializarse y complicar su estado de salud.

Quienes tienen seguridad social viven con la preocupación de perderla y no contar con el acceso a la atención médica y al tratamiento. Por otro lado, dos participantes del estudio siguen sin acceso a la seguridad social. Uno de ellos vive día a día la incertidumbre ante la falta de recursos materiales, ya que se vio obligado a dejar su trabajo a causa de la diálisis. Su preocupación permanente gira en torno a cómo conseguir los recursos para acceder a las consultas médicas, comprar las bolsas de diálisis y los materiales que necesita para el tratamiento, entre otros. $Y$ aunque ha hecho intentos de trabajar lavando automóviles, su estado de salud no le permite tener un trabajo estable. Comenta al referirse a tal asunto:

"Yo estaba aciegado con mi familia, aciegado con el dinero, ¿Dónde me iba a internar? ¿Dónde me iba a atender? Andaba muriéndome... Me decían los doctores cuando me veían '¿No has venido, 'eda?', 'La neta no tengo con qué. La neta, sin feria la caja no te atiende'... Por eso me iba a lavar (autos) y me iba bien. 'Ahora sí', dije, 'tengo para el internado, tengo para mis estudios, ahora sí tengo para esto'. Pero se te acaba, se te acaba en nefro".

El apoyo social tiene un papel central en el manejo de esta fuente de incertidumbre. Gracias a la familia, los amigos, los profesionales de la salud y las asociaciones civiles que los apoyan consiguen continuar con su tratamiento.

\section{Un futuro incierto}

Al experimentar cambios en el cuerpo y constatar la reconfiguración de su identidad y sus roles, el futuro les parece incierto e impredecible. Al referirse a sus metas a futuro, mencionan que no suelen pensar en eso. Enfatizan que sus metas no se han cumplido a partir de la aparición de la enfermedad. Por ahora, la meta de todos ellos es trasplantarse, pensando que así realizarán sus proyectos personales como formar una familia, trabajar o estudiar. Pero al mismo tiempo, la posibilidad de 
obtener el trasplante en un futuro cercano también es un tema que les causa incertidumbre. Principalmente para aquellos que no tienen un donador de vivo, debido a que las listas de espera para un órgano cadavérico son largas.

El manejo de la incertidumbre que les causa el futuro consiste básicamente en la realización de una serie de medidas de tipo religioso argumentando que solo "Él (Dios) sabe qué va a pasar". Otros se centran en el aquí y ahora, en disfrutar cada día, valorar lo que tienen y enfocarse en su tratamiento y en la búsqueda del trasplante. Prefieren postergar sus proyectos y planes a futuro una vez habiendo logrado el trasplante. También les es importante mantenerse ocupados para no pensar en ello, mantener la esperanza y la buena actitud.

\section{La incertidumbre médica}

\section{Información insuficiente}

Desde que aparecen los primeros síntomas de la enfermedad y les hacen el diagnóstico, los jóvenes sienten la necesidad de tener información sobre lo que les está pasando y lo qué les espera en el futuro. En estas circunstancias, la falta de información sobre la enfermedad y la diálisis también se vuelve una fuente de incertidumbre.

Al inicio, cuando están hospitalizados, ninguno parece tener idea de lo que les sucede. Inclusive después de recibir el diagnóstico, a excepción de los casos donde alguna persona cercana a ellos padeció esta enfermedad, no saben qué es la enfermedad renal. Y aunque los profesionales les dan el diagnóstico, no les explican a detalle en qué consiste y de qué manera puede afectar a su vida.

Para aquellos que inician el tratamiento inmediatamente después del diagnóstico surgen diversas incertezas desde el momento de la cirugía para la instalación del catéter, ya que hay casos en los que no saben para qué es y que puede ser permanente. Luis narra su experiencia cuando lo llevan a la cirugía del catéter:

"Llega un camillero y me llevan... me dice "¿ya te dijeron qué te van a hacer?", dije "no", "ah, te vamos a poner un catéter".... Yo me quedé (pensando) "Pero explíquenme qué es" y nada más me dice "te vamos a anestesiar"...Y es lo único que piensas "¿Qué me van a hacer?" 0 "¿qué tanto voy a tener que pasar? ¿Cuánto tiempo me lo van a dejar?... Y tú no sabes ni siquiera qué es eso, tú no sabes de todo eso y piensas bien muchas cosas".
Al darse cuenta de que la información que les brindan los profesionales es insuficiente, deciden buscar otras fuentes. Conversan con sus pares sobre cómo es vivir en tratamiento de diálisis, las asociaciones civiles les resuelven sus dudas y obtienen información por medio del internet, especialmente en los grupos de Facebook creados por las mismas personas enfermas.

\section{El tratamiento}

También cuando tienen información aumentan las dudas, ya que se cuestionan qué va a pasar con ellos. Sus incertezas y preocupaciones giran en torno al catéter, a los cuidados que implica y al nuevo estilo de vida que deben adoptar.

Quienes están en pre diálisis antes de iniciar la DPCA se informan sobre las terapias de reemplazo renal, lo que les causa incertidumbre al pensar en el momento que iniciarán la diálisis. Especialmente aquellos que han visto las complicaciones de salud de otras personas. Por ello evitan el inicio del tratamiento, ya que terminan asociándolo a mayores problemas de salud y la muerte. Un ejemplo, es el caso de Antonio, quien decide buscar tratamientos alternativos como son los medicamentos naturistas, homeopatía, acupuntura y diálisis oral. Sin embargo, su salud se complica hasta que después de varios meses decide ir a urgencias para que lo dialicen.

El manejo de este tipo de incerteza consiste principalmente en dos acciones: aceptar que la DPCA es necesaria para sobrevivir y mantener la esperanza y una buena actitud al respecto.

\section{El manejo del tratamiento}

La DPCA se realiza diariamente en casa, por tal motivo, la persona enferma y su familia es capacitada y se responsabiliza del tratamiento, lo que se convierte en fuente de incertidumbre ya que no saben si tomarán las mejores decisiones y cuidados.

Cuando realizan por primera vez los cambios de bolsa de diálisis en sus hogares dudan si lograrán hacerlo de forma correcta. Esta situación les causa preocupación y temor, en especial después de escuchar las numerosas recomendaciones de los profesionales de salud y constatar que otras personas han presentado peritonitis. Por ejemplo, para Antonio fue una situación complicada la primera diálisis:

"Sentía mucho miedo. Ahí te asustan de más, porque te dicen las cosas muy extremas... que hasta los movimientos tienes que cuidarlos, que no puedes hacer 
esto... Tenía miedo de contaminarme, de todo lo que me decían de la peritonitis, que te salía sangre, que te andabas muriendo casi casi, y pues sí, uno acaba asustado."

Suelen evitar lugares donde se expongan a un golpe 0 una infección, sin embargo, consideran que no es necesario cumplir con puntualidad los horarios de la diálisis, aunque creen importante realizar el número de cambios correspondiente.

La alimentación, hacer esfuerzos físicos o exponerse a otro tipo de enfermedades son temas que también les preocupan. Aunque saben que deben cuidar lo que comen les resulta difícil hacerlo, por lo que en algunos momentos no cumplen con la dieta sugerida aun sabiendo del riesgo que están tomando.

A pesar de los cuidados y decisiones que toman a lo largo del tratamiento, intentan pensar en que pueden hacer su vida tal como lo hacían antes. Se enfocan principalmente en y el seguimiento de las indicaciones médicas, pero mientras se sientan bien, pueden ser más flexibles en sus cuidados.

\section{Progreso potencial de la enfermedad}

Los jóvenes saben que pueden desarrollar otras enfermedades o complicaciones de salud, sea a causa del padecimiento o del tratamiento, lo que los hace sentirse inseguros por el potencial progreso de la enfermedad.

Uno de los casos es cuando los análisis médicos presentan datos de posibles problemas. Un ejemplo es el caso de Ana quien, por más que intenta seguir la dieta y el tratamiento, en los últimos estudios observa que tiene el fósforo alto. Esta situación la hace sentir preocupada:

"Pero no quiero que el día de mañana se llegue eso, que ésta enfermedad me lleve a otra cosa y a otra y otra. Sé que nuestro cuerpo se deteriora y pues nos duele una cosa y nos duele otra y es por la enfermedad".

La falta de control sobre la enfermedad los lleva a pensar en posibles complicaciones futuras, y se dan cuenta de que, aun cumpliendo con las indicaciones médicas, no pueden detener el progreso de la enfermedad.

Para manejar la incertidumbre por el progreso de la enfermedad, algunos buscan el trasplante para detener el desgaste físico en su cuerpo. A quienes les es difícil obtener un trasplante, han decidido disfrutar su día a día y creen que lo mejor es no pensar en el progreso de la enfermedad. Sin embargo, hay otros que quieren tener información sobre las complicaciones que puedan presentar.

\section{Discusión}

El objetivo de este trabajo fue comprender las experiencias de incertidumbre de jóvenes en tratamiento de DPCA. Hemos identificado dos modalidades de incertidumbre, la personal y la médica. Nuestros hallazgos también dan cuenta de las fuentes de la incertidumbre y las estrategias que llevan a cabo los participantes para su manejo.

La falta de recursos materiales destaca como una fuente de incertidumbre, tema que es de importancia en un contexto como el de México, donde gran parte de la población, no cuenta con protección social. Son pocos los estudios que hablan sobre ello8,21, donde reportan que los problemas económicos tienen efectos negativos como impedirles asistir a las consultas médicas, dejar de tomar medicamentos, abandonar el protocolo para trasplantes, entre otros.

Por otro lado, se observa cómo para las personas en DPCA, en medio de las incertezas y el sufrimiento, es importante mantener la esperanza y el apoyo de otras personas. Otros trabajos reportan resultados similares donde dicen que se muestran agradecidos y con esperanza, disfrutando el día a día y minimizando las cosas negativas ${ }^{22-24}$. Además, dicen que la obtención de la información y el apoyo de diversos actores es de suma importancia para ellos, especialmente el apoyo de la familia y de los profesionales de la salud ${ }^{13}$.

Cabe resaltar la importancia que tienen las redes sociales virtuales como fuente de información. Mercado-Martínez y Urias-Vázquez ${ }^{25}$ describen cómo las personas con ERC utilizan las redes virtuales para obtener de información, y que esto genera conocimientos complementarios 0 en algunas ocasiones hasta diferentes de los que brindan los profesionales.

La búsqueda del trasplante es central, viéndolo como la solución a su problema y una posibilidad de volver a la normalidad. Sin embargo, esto no es posible aún después del trasplante ${ }^{19}$, por lo que sería de interés profundizar con más detalle en las experiencias de incertidumbre después del trasplante.

Este trabajo tiene algunas limitaciones. Al ser un estudio realizado solamente con jóvenes y no hacer una 
comparación con las experiencias de otros grupos etarios, no se puede decir que estas experiencias de incertidumbre sean compartidas por todas las personas en diálisis. Además, hace falta profundizar en cómo manejan la incertidumbre.

Finalmente se concluye que son constantes las experiencias de incertidumbre que viven los jóvenes que utilizan el tratamiento de DPCA. Dichas experiencias tienen diferentes causas, algunas más personales, relacionadas con las alteraciones que el padecimiento y el tratamiento provocan en su cuerpo y su vida social. Mientras que otras están relacionadas con los cuidados que reciben, o la falta de calidad de los mismos, y la progresión de la enfermedad. La forma en que manejan la incertidumbre es influenciada por los recursos emocionales y materiales con los que cuentan, así también, por el apoyo que reciben de sus redes sociales. Cabe resaltar que desarrollan habilidades para enfocarse en múltiples alternativas y posibilidades, donde aprecian la fragilidad de sus situaciones de vida. Todo ello influye directamente en el manejo que hacen de su tratamiento $\mathrm{y}$ de sus relaciones interpersonales.

El reconocimiento de esas experiencias permitirá una nueva visión de atención profesional que no considere solamente las alteraciones físicas, sino también, los aspectos emocionales, sociales y culturales que están implícitos en la experiencia de una condición crónica, abriendo un dialogo más horizontal entre los jóvenes con ERC y el equipo de salud.

\section{Agradecimientos}

Expresamos nuestro más profundo agradecimiento a todos los participantes del estudio. Así también, a Francisco Javier Mercado Martínez quien como investigador y amigo supervisó y apoyó en el desarrollo del presente trabajo. Lamentamos su pérdida y agradecemos el legado que nos ha dejado, en lo académico y lo personal.

Recibido: 15-12-19

Revisado: 25-01-20

Modificado: 15-02-20

Aceptado: $30-02-20$

\section{Bibliografía}

1. Glassock R, Warnock D, Delanaye P. The global burden of chronic kidney disease: estimates, variability and pitfalls. Nat Rev Nephrol. 2017;13(2):104-14.

2. Lozano R, Gómez-Dantés H, Garrido-Latorre F, Jiménez-Corona A, Campuzano-Rincón JC, Franco-Marina $F$, et al. La carga de enfermedad, lesiones, factores de riesgo y desafíos para el sistema de salud en México. Salud Publica Mex, 2013;55:580-94.

3. Valdez-Ortiz R, Navarro-Reynoso $F$, Olvera-Soto MG, Martin-Alemañy G, Rodríguez-Matías A, Hernández-Arciniega $C R$, et al. Mortality in patients with chronic renal disease without health insurance in Mexico: opportunities for a national renal health policy. Kidney Int Rep, 2018;3(5):1171-82.

4. Li PKT, Chow KM, Van de Luijtgaarden MW, Johnson DW, Jager K., Mehrotra R, et al. (2017). Changes in the worldwide epidemiology of peritoneal dialysis. Nat Rev Nephrol, 2017; 13(2):90-103.

5. Tong A, Morton R, Howard K, McTaggart S, Craig JC. "When I had my transplant, I became normal." Adolescent perspectives on life after kidney transplantation. Pediatr Transplant. 2011;15(3):285-93.

6. Nicholas DB, Picone G, \& Selkirk EK. The lived experiences of children and adolescents with end-stage renal disease. Qual Health Res. 2011; 21(2):162-73.

7. Lewis $\mathrm{H}$, Arber $\mathrm{S}$. The role of the body in end-stage kidney disease in young adults: Gender, peer and intimate relationships. Chronic IIIn. 2015;1(3):184-97.

8. Díaz-Medina BA, Mercado-Martínez FJ. Obstáculos y estrategias de afrontamiento en la atención renal: estudio cualitativo en jóvenes con enfermedad renal crónica en diálisis peritoneal. Saúde Soc. 2019;28:275-86.

9. Conrad, P. Qualitative research on chronic illness: a commentary on method and conceptual development. Soc Sci Med. 1990; 30(11):1257-63.

10. Kuang K, Wilson SR. A meta-analysis of uncertainty and information management in illness contexts. $J$ Commun. 2017;67(3):378-401. 
11. Mishel M. Uncerainty in illness. J Nurs Scholarsh. $1998 ; 20(4): 225-32$.

12. Penrod J. Advancing uncertainty: untangling and discerning related concepts. Int $\mathrm{J}$ Qual Methods, 2002;1(4):54-61.

13. Tong A, Lesmana B, Johnson D, Wong G, Campell $D$, Craig JC. The perspectives of adults living with peritoneal dialysis: Thematic synthesis of qualitative studies. Am J Kidney Dis. 2013;61(6):873-88.

14. Curtin RB, Johnson HK, Schatell D. The peritoneal dialysis experience: insights from long-term patients. Nephrol Nurs J. 2004; 31(6):615-24.

15. Baillie J, Lankshear A. Patient and family perspectives on peritoneal dialysis at home: findings from an ethnographic study. J Clin Nurs. 2015;24(12):222-34.

16. Tong A, Morton R, Howard K, McTaggart S, Craig J. "When I had my transplant, I became normal." Adolescent perspectives on life after kidney transplantation. Pediatr Transplant. 2011;15(3): 285-93.

17. Mercado FJ. Entre el infierno y la gloria. La experiencia de la enfermedad en un barrio urbano. Guadalajara, México: Universidad de Guadalajara; 1996.

18. Lindseth A, Norberg A. Phenomenological hermeneutical method for researching lived experience. Scand J Caring Sci. 2004;18(2):145-53.

19. Martin SC, Stone AM, Scott AM, Brashers DE. Medical, personal, and social forms of uncertainty across the transplantation trajectory. Qual Health Res. 2010; 20 (2):182-96.
20. Brashers DE, Neidig JL, Russell, JA, Cardillo LW, Haas SM, Dobbs LK, Garland M, McCartney B, Nemeth, S. The medical, personal, and social causes of uncertainty in HIV illness. Issues Ment Health Nurs. 2003;24(5):497-522.

21. Mercado-Martínez $F$, Hernández-Ibarra $E$, Ascencio-Mera C, Díaz-Medina B, Padilla-Altamira C, Kierans C. Viviendo con trasplante renal, sin protección social en salud: ¿Qué dicen los enfermos sobre las dificultades económicas que enfrentan y sus efectos?. Cad Saude Publica. 2014;30:2092-100.

22. Santos FK, Valadares GV. Conhecendo o mundo do ser que enfrenta a diálise peritoneal: nexos simbólicos presentes no cotidiano. Revista Enfermagem UERJ. 2011;19(3):473-8.

23. Knihs ND, Sartori DL, Zink V, Roza BD, Schirmer J. A vivência de pacientes que necessitam de transplante renal na espera por um órgão compatível. Texto Contexto Enferm. 2013;22(4): 1160-8.

24. Lopes SG, Silva DM. Narratives of women on hemodialysis: waiting for a kidney transplant. Texto Contexto Enferm. 2014, 23(3):680-7.

25. Mercado-Martínez FJ, Urías-Vázquez JE. Enfermos renales hispanoamericanos en la época de las redes sociales virtuales: análisis de contenido de sus publicaciones, 2010-2012. Rev Panam Salud Publica. $2014 ; 35(5 / 6): 392-8$.

Este artículo se distribuye bajo una Licencia Creative Commons Atribución-NoComercial 4.0 Internacional. https://creativecommons.org/licenses/by-nc/4.0/

Open Access (C) (1) (8) 